\title{
A magnetosome chain viewed as a bio-elastic magnet
}

\author{
Ariel G. Meyra, ${ }^{a}$ Guillermo J. Zarragoicoechea ${ }^{a b}$ and Victor A. Kuz ${ }^{a}$
}

In light of the coarse-grained Monte Carlo numerical simulation method, the magnetosome chain stability of magnetotactic bacteria is analysed and discussed. This discrete chain of magnetic nanoparticles, encapsulated in a lipid membrane and flanked by filaments, orients bacteria in the geomagnetic field as a compass needle. Each magnetosome is a magnetite or greigite nanocrystal encapsulated in a soft lipid shell. This structure is modelled by a hard core with a magnetic dipole embedded and a cloud of electric dipoles which are able to move and rotate over the magnetic spherical core. In the present paper, some of the many possibilities of the model by varying the control parameters of the system are explored. Magnetic particles arrange in long linear clusters when the coating is removed. However, linear but twisted chains of magnetic particles emerge when there are electric dipoles in the coating shell. A unique linear and straight chain is not observed in any 3D

Received 16th December 2015, Accepted 5th April 2016

DOI: $10.1039 / c 5 c p 07795 h$

www.rsc.org/pccp numerical simulation; this result is in agreement with a real living system of bacteria in a geomagnetic field when proteins that form the filament are absent. Finally, the stability and magnetization of a magnetosome chain of 30 beads in one dimension set up are discussed resembling a real chain. The results suggest that a magnetosome chain not only orients bacteria but also should be considered as a potential storage of elastic energy.

\section{Introduction}

Bacteria use magnetosome chains to orient and move in the geomagnetic field to reach suitable sites of low oxygen concenmagnetic microstructure of magnetotactic bacteria is nowadays an active field of research. Magnetosomes are magnetic nanocrystals, ${ }^{1}$ constrained by invaginations of cell membranes, flanked by cytoskeletal filaments, and magnetically organized field in the range of 300-450 Oersted. ${ }^{2}$

The magnetic core, with a diameter between 20 and $50 \mathrm{~nm}$, could be composed of a nanoparticle of magnetite $\left(\mathrm{Fe}_{3} \mathrm{O}_{4}\right)$, greigite $\left(\mathrm{Fe}_{3} \mathrm{~S}_{4}\right)$, or several forms of iron sulphide, ${ }^{3,4}$ coated with a 3-5 nm thick lipid bilayer ${ }^{2}$ (phospholipids, fatty acids, and transmembrane proteins ${ }^{5}$ ).

If magnetosomes are not linked to the cytoskeleton, they do not self-organize into a straight chain. ${ }^{6}$ Biology plays an antientropy role in the self-organization of magnetosomes into a regular chain (an effective compass needle behaviour), avoiding spontaneous agglomeration in rings, spherical clusters, etc. ${ }^{7-11}$

${ }^{a}$ IFLYSIB, 59 789, La Plata, Argentina. E-mail: vasco@iflysib.unlp.edu.ar;
There is also an interesting fact that bacteria do not agglomerate, they only react to the geomagnetic field. ${ }^{12}$

In this work a reductionist model to describe the structure of the magnetosome is presented, which considers a hard core with a magnetic dipole embedded and a set of electric dipoles to represent the magnetosome membrane.

The chain stability, in three dimensions (3D) and in one dimension (1D), is analysed for different values of the respective dipoles, and also under the effect of an external magnetic field. We keep in mind in detail the 1D case which strongly resembles the magnetosome chain. It is shown that these kinds of biomagnetic structures have non-linear elastic properties which could be used as alternative sources ${ }^{13}$ of nanobot propulsion.

\section{The model}

Magnetosomes are represented by $N$ magnetic spheres of radius $R_{\mathrm{s}}$, coated with a constant number $n$ of dipolar particles. Each sphere has a magnetic dipole moment $\mu_{\mathrm{m}}$ as indicated in Fig. 1, whilst a dipolar particle has an electric dipole moment $\mu_{\mathrm{e}}$, with $\sigma$ being its diameter. The total number of electric dipoles (EDs) in each simulation box is $N n$. The quantities $\sigma$ and $\mu_{\mathrm{e}}$ are taken as reference to scale lengths and energies. ${ }^{14-16}$ Simulations in $3 \mathrm{D}$ are performed in a cubic box of side $100 \sigma$ with a cut-off radius of $50 \sigma .{ }^{13,14}$ EDs are restricted to move over 
Fig. 1 Spheres are coloured to indicate the spacial orientation of magnetic and electric dipoles. $R_{\mathrm{s}}$ is the radius of two magnetic nanospheres and $\delta$ is the distance between the dipolar particle and the surface of the sphere. It could be associated with a nonpolar tail of a zwitterionic surfactant or any hydrophobic-hydrophilic molecule. $\theta, \vec{R}_{i j}$, and $\vec{r}_{k l}$ are the tilt angle, the centre-centre distance between two magnetic spheres, and the distance between any two electric dipoles, respectively.

a sphere of radius $R_{\mathrm{s}}+\delta$, being $\delta>0$. This restriction represents a generic surfactant or phospholipid molecule with a non-polar tail of length $\delta$ linked to the surface of the sphere, and a polar head with an electric dipole $\mu_{\mathrm{e}}$ (see Fig. 1).

The ED tilt angle $\theta$ is formed between the unit vector determining the ED orientation, $\vec{s}_{i}$, and the vector normal to the surface of the sphere. It might represent the flexibility between the hydrophobic tail and the hydrophilic head of the surfactant molecules or proteins. The $N$ spheres plus the $N n$ dipolar particles are able to move within the box. EDs follow the translational and rotational motions of the sphere they are attached to (relative position and orientation constant). Then each electric dipolar particle could be displaced and rotated on the surface of its sphere. A parallel tempering technique has been applied to the system in order to decrease the simulation time, as well as periodical boundary conditions. ${ }^{14,15}$

Magnetic nanoparticles (MNs) interact via a hard core repulsion plus a magnetic dipolar potential: ${ }^{10,11,17,18}$

$$
\begin{aligned}
U_{\mathrm{m}} & \left(\vec{R}_{i j}, \vec{m}_{i}, \vec{m}_{j}\right) \\
= & \begin{cases}\infty & \text { if } \quad R_{i j} \leq 2 R_{\mathrm{s}} \\
\frac{\mu_{\mathrm{m}}{ }^{2}}{R_{i j}{ }^{3}}\left[\left(\vec{m}_{i} \cdot \vec{m}_{j}\right)-\frac{\left.3\left(\vec{m}_{i} \cdot \vec{R}_{i j}\right)\left(\vec{m}_{j} \cdot \vec{R}_{i j}\right)\right]}{R_{i j}{ }^{2}}\right] & \text { if } \quad R_{i j}>2 R_{\mathrm{s}}\end{cases}
\end{aligned}
$$

The interaction between EDs is also represented by a hard core repulsion and a dipolar pair potential, ${ }^{14,16}$ as follows:

$$
U_{\mathrm{e}}\left(\vec{r}_{k l}, \vec{s}_{k}, \vec{s}_{l}\right)= \begin{cases}\infty & \text { if } \quad r_{k l} \leq \sigma \\ \frac{\mu_{\mathrm{e}}^{2}}{r_{k l} l^{3}}\left[\left(\vec{s}_{k} \cdot \vec{s}_{l}\right)-\frac{3\left(\vec{s}_{k} \cdot \vec{r}_{k l}\right)\left(\vec{s}_{l} \cdot \vec{r}_{k l}\right)}{r_{k l}{ }^{2}}\right] & \text { if } \quad r_{k l}>\sigma\end{cases}
$$

Though we have done MC simulation (equilibrium state), it is appropriate to say that in this system with slow dynamics, the cross-interaction terms in the hamiltonian (electric dipolemagnetic field, magnetic dipole-electric field) are not significant because the ratio between the dipole velocity and the speed of light is much less than $1 .^{21}$

Magnetic and electric dipole orientations are defined by $\vec{m}$ and $\vec{s}$, respectively and $\vec{R}_{i j}=\vec{R}_{i}-\vec{R}_{j}$ and $\vec{r}_{k l}=\vec{r}_{k}-\vec{r}_{l}$ are the distance between any two MNs or EDs, respectively, within the simulation box. It must be remarked that the distance between any ED and any MN is never less than $R_{\mathrm{S}}+\delta$ (see Fig. 1), being $R_{\mathrm{S}}=4.5 \sigma$ and $\delta=1.5 \sigma$. Parallel tempering simulation is applied to 10 replicas of the statistical system at temperatures defined as $T_{i}^{*}=k T_{i} \sigma^{3} / \mu_{\mathrm{e}}{ }^{2}(i=1,10)$, with $T_{i}^{*}$ being the temperature of the replica $i$ and $k$ being Boltzmann's constant, but analysis is done only for those systems at the reference temperature $T^{*}=$ $k T \sigma^{3} / \mu_{\mathrm{e}}{ }^{2}=0.25$, with $\mu_{\mathrm{m}}{ }^{*}=50$. These dimensionless values are representatives of a real magnetosome system when parameters are in the following ranges: $20-50 \mathrm{~nm}$ for the magnetite radius, 2-5 $\mathrm{nm}$ for the protein shell, and 3.8-4.2 $\times 10^{-17} \mathrm{~A} \mathrm{~m}^{2}$ (proportional to the MN's volume ${ }^{22}$ ), and $2.0-3.0 \times 10^{-28} \mathrm{C} \mathrm{m}$ for magnetic and electric dipole moments, respectively. Temperatures are in the range of $4-30{ }^{\circ} \mathrm{C}$, and the earth's magnetic field is in the range of $0.25-0.65$ Gauss.

For the equilibration we have done $2 \times 10^{6} \mathrm{MC}$ steps, and 5 $\times 10^{5}$ MC steps for production. Each step consists, for every replica, of a random movement of (a) each magnetic sphere, with the electric dipole particles frozen on its surface, and (b) each electric particle over the surface of the sphere to which it is attached to. A particle movement, magnetic or electric, consists of a simultaneous displacement and rotation of the particle. Trails are accepted or rejected according to the Boltzmann probability distribution, with acceptance ratios on the order of $50 \%$ for both particle movements. Swaps between adjacent replicas, which are chosen randomly, are attempted each $5 \times$ $10^{2}$ steps. ${ }^{19,20}$ With the temperature scale defined above an averaged acceptance ratio of $40 \%$ is obtained. Simulations are initiated with randomly distributed electric dipoles and magnetic spheres.

\section{Results and discussion}

As stated previously, magnetic and electric dipoles represent a single-domain magnetite crystal and its coating lipid bilayer and proteins, respectively. This set is called a bio-magnetic particle (BMP). If a BMP loses its lipid bilayer or the envelope membrane then it becomes a pure MN. A first comparative analysis is done on three different 3D systems (particles are randomly distributed in a cubic box of size $L=100 \sigma$ ). Simulated systems are: (1) $30 \mathrm{MNs}$, (2) 30 BMPs, and (3) 30 BMPs with an external magnetic field applied (see Fig. 2a, b and c, respectively). Cluster analysis is done with the help of the radial distribution function (RDF) of magnetic nanospheres. ${ }^{14,15}$

Cluster structures of MNs and BMPs (see Fig. 2a and b) are rather similar, they are mainly chain-like, but they are longer in the case of MNs (see Fig. 2a). In both cases the magnetization is null. RDFs have a main peak for the three systems studied, for MNs the peak is at $R_{i j} \approx 2 R_{\mathrm{s}}$, but for BMPs it is at $R_{i j} \approx 2\left(R_{\mathrm{S}}+\delta\right)$ (see Fig. 3). It resembles to those found by Komeili et al. ${ }^{6}$ When 
an external magnetic field is applied to a BMP system (Fig. 2c), a smooth peak at $R_{i j} \approx 2\left(R_{\mathrm{S}}+\delta\right)$ appears. The self-assembly of

These theoretical results are in qualitative agreement with those obtained by using electron microscopic evaluation of magnetosomes liberated from cells. ${ }^{23}$ Along the same line of

Fig. 2 Snapshots of the systems (1), (2) and (3), mentioned in the text, with $T^{\star}=0.25$ and $0^{\circ} \leq \theta \leq 45^{\circ}$.

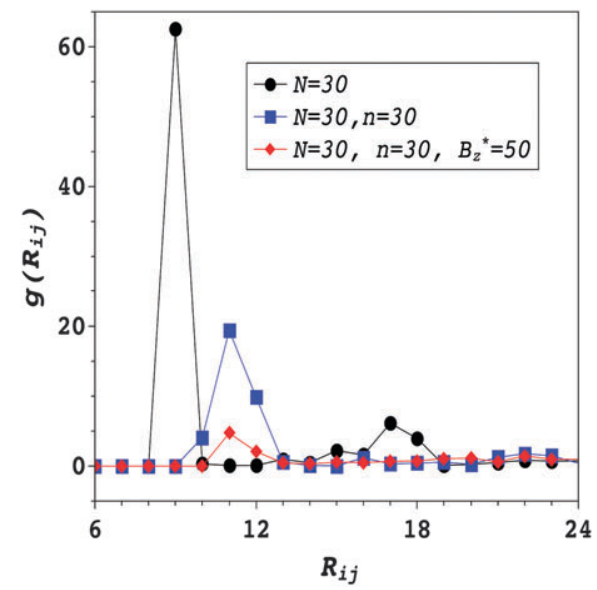

Fig. 3 Radial distribution functions for three simulated systems. facts Philipse $e t$ al. ${ }^{24}$ showed a selection of cluster morphologies for magnetite colloids extracted from cells. Images (transmission electron micrograph) are similar to those obtained in the present work. In any case those topological organizations of BPMs are induced as a consequence of the minimization of the total energy of the system. It is worth remarking that the cluster size is never bigger than 8 nanospheres, being larger for the MNs (see Fig. 2a).

As our purpose is to inquire into the straight chain of magnetosome formation and stability, 1D simulations are performed and analysed. This set up mimics a magnetotactic bacterium as a needle compass. The simulation was done for different values of magnetosome linear densities. The number of nanospheres, dipoles and temperatures were constant, being $N=30, n=30$ and $T^{*}=0.25$, respectively. The range of linear densities $\rho=N / L=30 / L$ is bound by $400 \sigma \leq L \leq 1000 \sigma$.

Before discussing these results in light of some experimental studies on the subject, the dimensionless energy per BMP versus density (Fig. 5) is analysed for the 1D system with 400 $\leq L^{*} \leq 1000$, and it is compared with the same number of BMPs but in a 3D bulk system.

As can be seen in Fig. 4, when density decreases the BMP interdistance increases giving as a result a sparse BMP arrangement. The lowest potential energy is obtained in those systems with higher density (see Fig. 5). A closer analysis of this figure
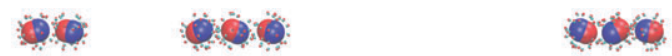

\section{oopo oopo poro}

Fig. 4 Two snapshots of the 1D system for different densities, at $T^{\star}=0.25$. The bottom snapshot represents the most dense system with $L=400 \sigma$, while the top one is for $L=900 \sigma$.

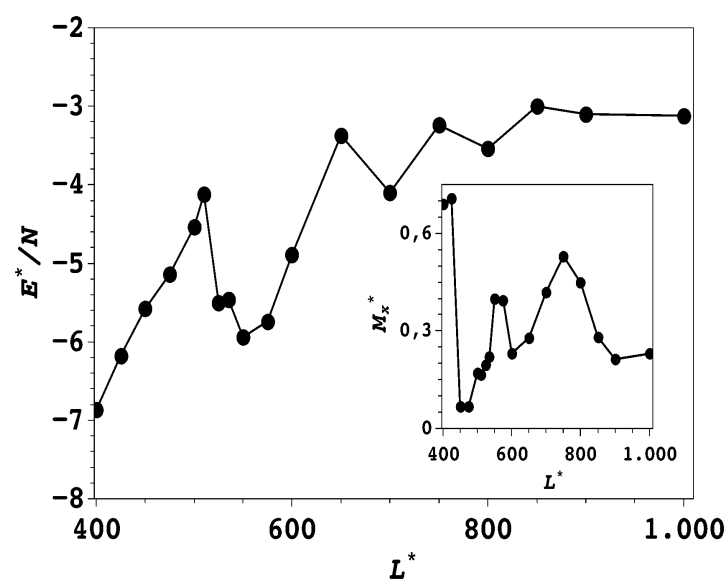

Fig. $5 E^{*} / N$ vs. inverse of the density, which is proportional to $L^{*}$ for a $1 D$ system, with $N=30, n=30, T^{\star}=0.25, \mu_{\mathrm{m}}{ }^{*}=50$, and $\mu_{\mathrm{e}}{ }^{*}=1$. In the inset, $M_{x}{ }^{*}$ is represented, which is the module of the component of the magnetization parallel to the axis of the 1D system, vs. linear density. 
1 reveals that at $L^{*}=400$ the potential energy is the lowest, which indicates that the most dense systems are the most stable. For the highest densities a maximum magnetization is also found (see the inset in Fig. 5), with $M_{x}^{*}=(1 / N) \mu_{\mathrm{m}}^{*}\left|\sum_{i=1}^{N} \vec{m}_{i} \cdot \vec{e}_{x}\right|$, it might indicate that part of the stability of the system is due to magnetic attraction among BMPs. But, as $L^{*}$ increases, energy linearly grows till $L^{*} \approx 510$ (a local maximum) then sharply decreases to a relative minimum at $L^{*} \approx 550$. This second minimum is due to the electric dipole interaction. For lower densities, with $700<L^{*} \leq 1000$, energy oscillates till a saturation value. This is an important result from the model, and it indicates that once the magnetosomes stick to the filaments, ${ }^{6,7}$ the system evolves decreasing its potential energy and increasing its density by forming a long dense and straight chain with the highest magnetization. Another interesting result is that the potential energy pattern of the system resembles a typical saw-tooth pattern found when elasticity in biological molecules or tissues is evaluated. ${ }^{25,26}$

In Fig. 6, the self-induced components of the electric and magnetic fields of a $1 \mathrm{D}$ system are analyzed. It is worth mentioning that components orthogonal to the main axis of the systems are at least one order of magnitude lower than the parallel one. An interesting result is that a local minimum in the magnetic field coincides with a local maximum in the electric field and vice versa. This opposite behaviour could be in fact a complementary one, the effect being larger for higher densities, $400<L^{*}<550$, which is coincident with the absolute maximum and minimum of the magnetization. The self-induced magnetic field follows the magnetization in terms of linear density (see the inset in Fig. 5) in this range of densities.

Now $E^{*} / N$ as well as $M_{x}^{*}$ is evaluated, for a 1D system when an external magnetic field parallel $B_{\|^{*}}=B_{x}{ }^{*}$ or transversal $B_{\perp}{ }^{*}=$ $B_{z}^{*}$ to the main axis is applied, which could be similar to the effect of the earth magnetic field over a magnetosome chain.
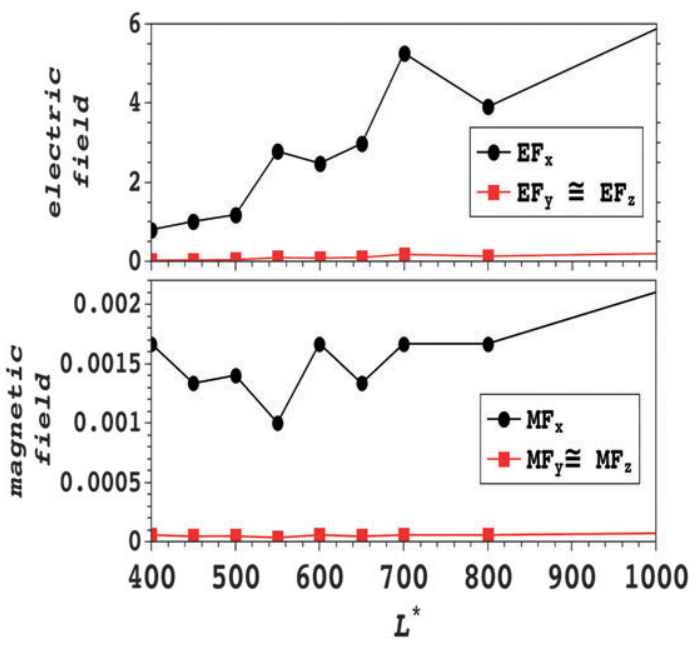

Fig. 6 Self-induced electric and magnetic fields for a 1D system of different linear densities.
The system is defined as follows: $L^{*}=400, N=30, n=30$, and $\mu_{\mathrm{m}}^{*}=50$ at $T^{*}=0.25$. The magnetic energy due to the external field is: $E^{*}=-\mu_{\mathrm{m}}{ }^{*} \vec{m} \cdot \vec{B}^{*} .^{27}$

When $B_{x}^{*}=B_{z}^{*}=0$ the system minimizes its potential energy aligning its magnetic dipoles in the preferential axis, $(x$ in this case), giving $M_{x}^{*} \approx 0.7$ (see the inset Fig. 8). $E^{*} / N$ remains nearly constant when the applied field is $0<B_{x}{ }^{*}<0.7$, but for $0.7<B_{x}^{*}<1.2$, the energy sharply decreases, becoming then linear (see Fig. 8). At small applied $B_{x}{ }^{*}$ magnetization decreases, and there is a clear competition between kinetic and potential energy (magnetic and electric dipolar contributions). For $B_{x}^{*} \approx 1 M_{x}^{*}$ abruptly increases $\left(M_{x}^{*} \rightarrow 1\right)$, and all BMPs are aligned under the effect of the field.

When the system is under the effect $B_{z}^{*}$ the situation is rather different. For $B_{z}^{*} \leq 0.2$ there is a sudden decrease in the potential energy while $M_{x}{ }^{*}$ slightly decreased. For $0.2 \leq B_{z}^{*} \leq 2$ potential energy remains nearly constant and for $B_{z^{*}} \geq 2$ it starts decreasing, while $M_{x}^{*}$ slowly decreases.

It can be noticed that for $B_{x}^{*}=B_{z}^{*} \approx 0.8, E^{*} / N$ and also $M^{*}$ are equal, but for $B_{x}^{*}=B_{z}^{*} \gg 0.8$ they are completely different. There is an extra potential energy when the bacterium is not aligned to the external field. This excess of energy could be elastically registered by protein filaments, this mechanism being the responsible for the bacterium orientation.

Now let us analyse our approach in light of some experimental results on the subject. The process of bacterial magnetosome formation seems to be as follows: magnetosome vesicle formation, arrangement of the vesicles in chains, iron uptake by the cell, iron transport into the magnetosome vesicle and controlled $\mathrm{Fe}_{3} \mathrm{O}_{4}$ (or $\mathrm{Fe}_{3} \mathrm{~S}_{4}$ ) biomineralization within the magnetosome vesicle. ${ }^{28}$ At this point let us comment the temporal order of our research: first we try to get a chain of BMPs in a 3D space and as we pointed out previously we got a short and twisted chain even if an important external magnetic field was applied to the system. Then, we set up a 1D nanosphere system $^{6,7}$ in order to test the mechanical stability for different densities and external perturbations (see Fig. 4). The results corroborate that a more dense chain is more stable, having lower energy and higher total magnetic moment.

In Fig. 9, the effect of temperature on different systems with and without an external magnetic field can be seen. Stability is more favorable in the less energetic 1D systems, with the energy smoothly increasing with temperature. An interesting result is found when a parallel or a tranversal magnetic field, $B_{x}^{*}=B_{z}^{*}=$
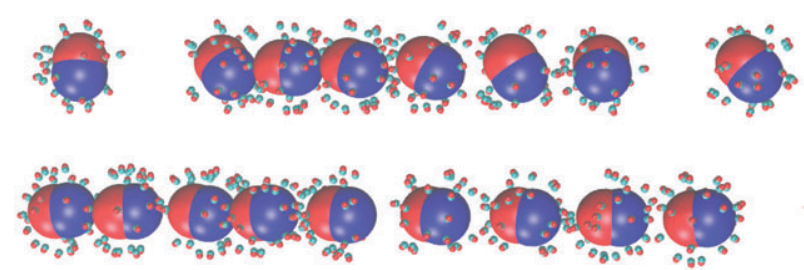

Fig. 7 Two snapshots of the 1D system when an external magnetic field $\left(B_{x}{ }^{*}=B_{z}{ }^{*}=5\right)$ is applied in the horizontal $x$ direction (bottom snapshot), or in the $z$ vertical direction (top snapshot).
1

10 15 
Fig. 8 Energy per sphere vs. external magnetic field, with $N=30, n=30$ $T^{*}=0.25$ and $\mu_{\mathrm{m}}{ }^{*}=50$ for a $1 \mathrm{D}$ system with $L^{*}=400$. In the inset $M_{x}{ }^{*}$ is shown.

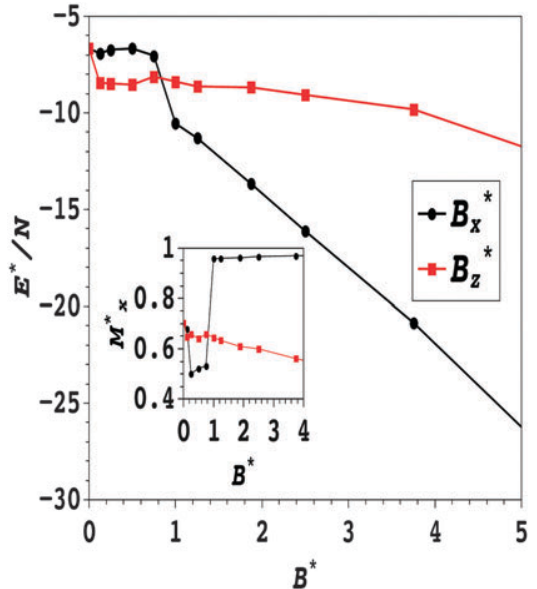

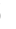

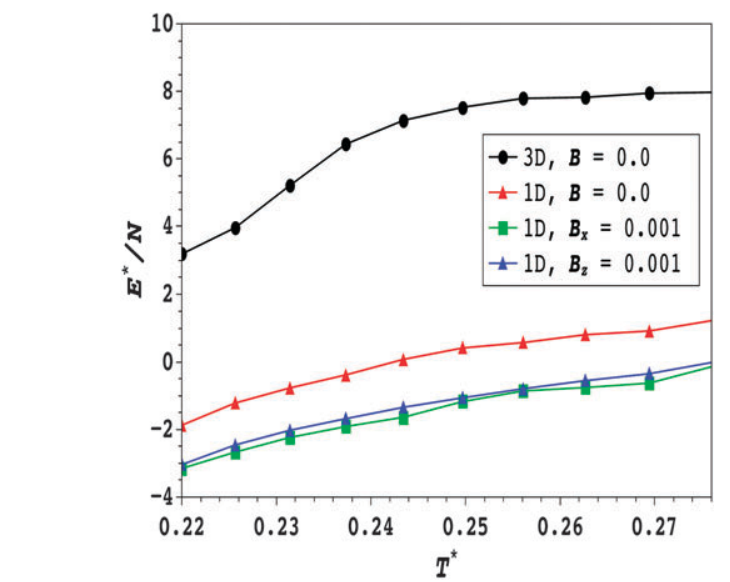

Fig. 9 Energy per sphere vs. dimensionless temperature, $N=30, n=30$, $\mu_{\mathrm{m}}{ }^{*}=50$, and $\mu_{\mathrm{e}}{ }^{*}=1$.

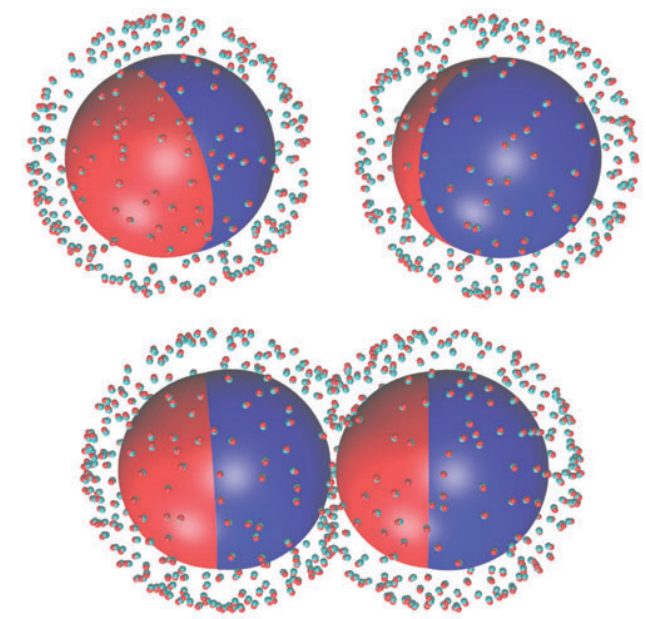

Fig. 10 Snapshots of the two sphere system at different distances, $N=2$, $n=300, \mu_{\mathrm{m}}{ }^{*}=300, \mu_{\mathrm{e}}^{*}=1$ and $T^{\star}=0.25$, when $R_{i j} \geq\left(2\left(R_{\mathrm{s}}+\delta\right)\right)$ (top) and $R_{i j}<\left(2\left(R_{\mathrm{s}}+\delta\right)\right)$ (bottom).

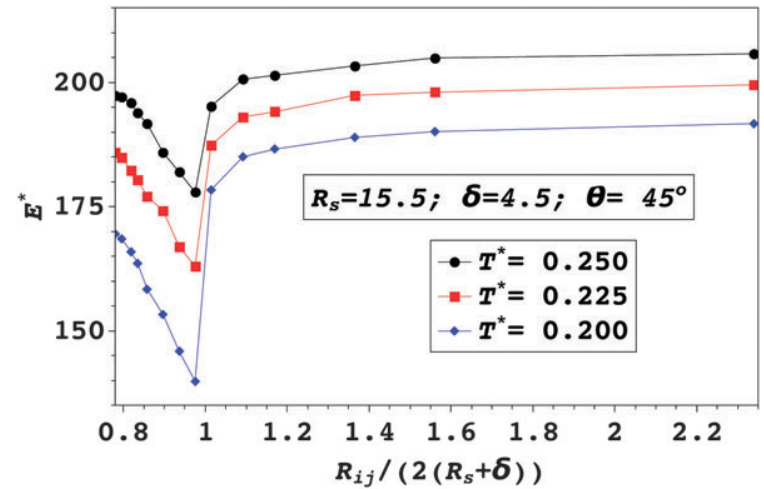

Fig. 11 Potential energy between two spheres for three different temperatures.

0.001 , in the order of the earth magnetic field, is applied to the 1D system. The subtle difference in energy between the two arrangements could allow the bacterium to explore its environment without being biased in a preferential direction.

Finally, the approaching of two coated nanospheres is simulated in order to estimate the potential energy for three different temperatures (see Fig. 10 and 11). The asymptotic behaviour comes from the dipolar interaction energy from the cloud of each nanosphere. As can be seen, at least in this range of temperatures, $E^{*}$ has an absolute minimum value. This behaviour could be an indication that the stability of the system is mainly due to the dipolar clouds that contribute to the chain cohesion and elasticity. The minimum in the energy is in agreement with the observed separation of nanoparticles in the magnetosome chain.,

\section{Conclusions}

Modelling a magnetosome chain as a magnetic dipole coated with a set of electric dipoles has been shown to be an interesting approach to disentangle how magnetosomes self-assemble.

According to our 3D Monte Carlo simulation, it is concluded that it is unlikely to obtain a unique and straight chain of magnetosomes, even under the effect of a soft or strong external magnetic field (see Fig. 3). A 1D system of coated nanospheres is perhaps an extremely reduced model to represent a BMP assembly. However, the simulation shows a very interesting result: among all the possible configurations, it seems that bacteria choose the one which consumes the lowest energy to assemble the magnetosomes with the benefit of having the lowest potential energy with the highest magnetic moment (see Fig. 4 and 5). When a magnetotactic bacterium crosses the earth magnetic field (see Fig. 7), there is a change in the magnetic energy and consequently an increment of the magnetosome elastic energy (Fig. 8). This energy, when released, might contribute to the bacterium's propulsion. A change in the relative position and/or orientation of the BMPs under the effect of an external magnetic field not only might save elastic energy but also may cause a structural rearrangement of electrons in proteins, lipids or in any biological 


\section{References}

in Prokaryotes, Microbiology Monographs, 2006, 2, 167-191.

2 R. E. Dunin-Borkowski, M. R. McCartney, R. B. Frankel, D. A. Bazylinski, M. Posfai and P. R. Buseck, Science, 1998, 282, 1860-1870.

3 D. Bazylinski, B. Heywood, S. Mann and R. Frankel, Nature, 1993, 366, 218.

4 D. Bazylinski, R. Frankel, B. Heywood, S. Mann, J. King, P. Donaghay and A. Hanson, Appl. Environ. Microbiol., 1995, 61, 3232-3239.

5 D. Bazylinski and R. B. Frankel, Nat. Rev. Microbiol., 2004, 2, 217-230.

6 A. Komeili, Z. Li, D. K. Newman and G. J. Jensen, Science, 2006, 311, 242-245.

7 A. Scheffel, M. Gruska, D. Faivre, A. Linaroudis, J. M. Plitzko and D. Schler, Nature, 2006, 440, 110-114.

8 D. Schuler, Arch. Microbiol., 2004, 181, 1-7.

9 A. Scheffel and D. Schuler, J. Bacteriol., 2007, 189, 6437-6446.

10 S. Knatorovich, R. Weeber, J. Cerda, C. Holm and J. Magnetism, J. Magn. Magn. Mater., 2011, 323, 1269-1272.

11 M. Klinkigt, R. Weeber, S. Kantorovich and C. Holm, Soft Matter, 2013, 9, 3535-3546.
12 R. B. Frankel, Biological Permanent Magnets, Hyperfine Interact., 2003, 151, 145-153.

13 R. Dreyfus, J. Baudry, M. L. Roper, M. Fermigier, H. A. Stone and J. Bibette, Nature, 2005, 437, 862-865.

14 M. P. Allen and D. J. Tildesley, Computer Simulations of Liquids, University Press, Oxford, 1987.

15 D. Frenkel and B. Smit, Understanding Molecular Simulation. From Algorithms to Applications, Academic Press, San Diego, 1996.

16 A. G. Meyra, V. A. Kuz and G. J. Zarragoicoechea, Mol. Phys., 2012, 110, 85-93.

17 J. H. Van Vleck, J. Chem. Phys., 1937, 41, 61-80.

18 J. Garcia-Otero, M. Porto, J. Rivas and A. Bunde, Phys. Rev. Lett., 2000, 84, 167-170.

19 A. Imperio and L. Reatto, J. Phys.: Condens. Matter, 2004, 16, S3769.

20 D. J. Earl and M. W. Deem, Phys. Chem. Chem. Phys., 2005, 7, 3910-3916.

21 A. Kholmetskii, O. Missevitch and T. Yarman, Eur. Phys. J. Plus, 2014, 129(10), 1-13.

22 M. de Sousa, M. Fernandez van Raap, P. Rivas, P. Mendoza Zelis, P. Girardin, G. Pasquevich, J. Alessandrini, D. Muraca and F. Sanchez, J. Phys. Chem. C, 2013, 117(10), 5436-5445.

23 Y. A. Gorby, T. J. Beveridge and R. P. Blakemore, J. Bacteriol., 1988, 170, 834-841.

24 A. P. Philipse and D. Maas, Langmuir, 2002, 18, 9977-9984.

25 T. E. Fisher, P. E. Marszalek and J. M. Fernande, Nat. Struct. Mol. Biol., 2000, 7, 719-724.

26 G. Bao and S. Suresh, Nat. Mater., 2003, 2, 715-725.

27 Y. Lalatonne, L. Motte, J. Richardi and M. P. Pileni, Phys. Rev. E: Stat., Nonlinear, Soft Matter Phys., 2005, 71, 011404.

28 R. B. Frankel and D. A. Bazylinski, Trends Microbiol., 2006, 14, 329-331.

29 K. Grunberg, E. C. Muller, A. Otto, R. Reszka, D. Linder, M. Kube, R. Reinhardt and D. Schuler, Appl. Environ. Microbiol., 2004, 70, 1040-1050.

$$
0
$$

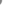

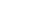

